\title{
Bioequivalence of Oral Products and the Biopharmaceutics Classification System: Science, Regulation, and Public Policy
}

\author{
KS Amidon ${ }^{1}$, P Langguth ${ }^{2}$, H Lennernäs ${ }^{3}, \mathrm{~L} \mathrm{Yu}^{4}$ and GL Amidon ${ }^{5}$
}

The demonstration of bioequivalence (BE) is an essential requirement for ensuring that patients receive a product that performs as indicated by the label. The BE standard for a particular product is set by its innovator, and this standard must subsequently be matched by generic drug products. The Biopharmaceutics Classification System (BCS) sets a scientific basis for an improved BE standard for immediate-release solid oral dosage forms. In this paper, we discuss $\mathrm{BE}$ and the $\mathrm{BCS}$, as well as the issues that are currently relevant to BE as a pharmaceutical product standard.

A recent report by Benet and Larregieu ${ }^{1}$ in this journal articulates issues, of both science and public policy, that currently surround the regulatory BE standard. That report ${ }^{1}$ focuses on the use of study results from metabolism, mass balance, and permeability studies and the $\mathrm{BCS}^{2,3}$ to set a $\mathrm{BE}$ standard for solid oral immediate-release dosage forms, i.e., the use of "biowaiver" applications that allow the waiver of an in vivo BE test. Here we discuss further issues pertaining to scientific evidence and regulatory requirements in $\mathrm{BE}$ testing. ${ }^{1}$

\section{BE TESTING PAST AND PRESENT}

BE, defined by the US Food and Drug Administration's (FDA's) Orange Book ${ }^{4}$ as the "display [of] comparable bioavailability when studied under similar experimental conditions," serves as a central scientific and regulatory standard for virtually all oral pharmaceutical products. ${ }^{5}$ The most recent regulatory statement on BE, the European Medicines Agency's "Guideline on the Investigation of Bioequivalence" (2010), defines the term similarly. ${ }^{6}$ The Orange Book further follows the language of the Drug Price Competition and Patent Term Restoration Act (Hatch-Waxman Act) of 1984: "Bioequivalent drug products are therapeutically equivalent and, therefore, interchangeable." Historically the in vivo matching of human systemic concentrations of active drug-and, when important for therapeutic efficacy, metabolite(s) - and comparison with a reference product (e.g., the Reference Listed Drug (product) ${ }^{4}$ ) has been the gold standard for BE and therapeutic interchangeability. This standard is widely accepted. However, the scientific question involved here is still open: what evidence should be required to support regulatory standards and decisions regarding BE?

\section{DEVELOPMENT OF THE BE STANDARD}

The science underlying $B E$ has been much discussed by the pharmaceutical industry, academics, and regulatory scientists since the 1960s. In the 1960s, Congress explored the idea of mandating, whenever possible, the prescribing of generic "drugs" (drug products). ${ }^{7}$ We note that the term "drug" appears frequently in these debates; in our opinion, the term is often ambiguous. In almost all contexts related to generic equivalence and BE testing, we interpret the term "drug" to be a reference to a drug product (as opposed to an "active pharmaceutical ingredient"). In regulatory and legislative language, this ambiguity reaches back as far as Section 6 of the Pure Food and Drug Act of 1906, which defines "drug" as "any substance or mixture of substances intended to be used for the cure, mitigation, or prevention of disease of either man or other animals." We believe that this conflation of the terms "drug" and "drug product" impedes our understanding even today.

Although Congress never implemented this generic substitution policy idea, BE rapidly revealed itself as a crucial scientific link between the extensive clinical efficacy testing required of innovator drug products (i.e., phase III testing) by the 1962 federal drug amendments and the marketed forms of those innovator products. ${ }^{8}$ The significance of $\mathrm{BE}$ in the development of innovator products and potential generic equivalents (i.e., therapeutically equivalent and interchangeable drug products) also quickly became clear.

Major scientific figures at the time recognized the significance of $\mathrm{BE}$ testing, if not the complexity of setting a regulatory standard. 
The pharmacologist Alfred Gilman, for example, wrote the "White Paper on the Therapeutic Equivalence of Generic Drugs" in 1969 for the Policy Advisory Committee of the National Academy of Sciences' Drug Efficacy Study. ${ }^{9,10}$ Gilman strongly stated that in vivo studies were necessary for the demonstration of equivalence. He argued that "all producers, and certainly the generic houses, should be required to submit proof of the performance of their drugs [presumably meaning drug products] in human patients before they are permitted to market them." ${ }^{10}$ Further investigation of BE led to a 1974 Office of Technology Assessment report, "Drug Bioequivalence," 8 that presented 11 conclusions and recommendations to Congress. Although the title of this influential report uses the term "Drug," the report focuses almost exclusively on drug products. The initial preliminary publication of a list of therapeutically equivalent drug products in the Federal Register in 1979 (the list became the first edition of the FDA Orange Book one year later (for the current edition, see ref. 4)), represents the first binding form of regulatory language based on $\mathrm{BE}$ research and testing. In the 1980s, after the Drug Price Competition and Patent Term Restoration Act of 1984 allowed generic drug firms to reference the data contained in innovator companies' new drug applications at the FDA, and allowed generic products to be marketed under essentially the same product label as the related innovator product, the political and business issues surrounding $\mathrm{BE}$ - and therefore the need for further scientific development of $\mathrm{BE}$ testing-became yet more acute.

Both the science and the regulatory policy with respect to $\mathrm{BE}$ have advanced significantly in the past 25 years. ${ }^{5,11}$ The value of the contribution made by the evolving science and practice of in vivo $\mathrm{BE}$ studies to ensuring the quality and performance of pharmaceutical products is unquestionable. The development of BCS-based science and regulation since the early 1990s has been another important element in this recent advancement of the understanding and implementation of BE. ${ }^{12}$ Further elaborations of BCS and the development of the Biopharmaceutics and Drug Disposition Classification System continue to expand the scientific and mechanistic understanding of $\mathrm{BE},{ }^{13,14}$ as well as molecular absorption, distribution, metabolism, and elimination. Although the relative bioavailability-based view of BE has received a substantial amount of scientific consideration since the 1970s (including study design, population sampling, and statistical evaluation ${ }^{11}$ ), BE remains a debated and inadequately developed approach to regulating pharmaceutical drug products. In particular, for oral products, the complex relationships between drug and drug product dissolution in vivo and the effects of excipients, intestinal permeation and transit, absorption, metabolism, excretion, and systemic availability continue to be much discussed. All these issues are important to the ongoing development of BE testing, both in vivo and in vitro. BE pertains to the same drug ("active pharmaceutical ingredient") in different products. Dissolution testing is currently central to in vitro BE testing, yet major scientific questions remain to be resolved: the feasibility of (i) using in vitro dissolution results to predict in vivo performance and (ii) using other data (e.g., absorption, metabolism, and, especially, tissue culture results) as a basis for setting in vitro dissolution and $\mathrm{BE}$ standards. Regulatory standards and practices for BE testing, particularly the BCS approach, have highlighted the relationships among permeability, absorption, metabolism, and drug dissolution of the product, in vitro and in vivo, and have established a scientific basis for setting regulatory in vitro $\mathrm{BE}$ dissolution standards for oral immediate-release products. ${ }^{2,5}$

\section{THE SCIENCE AND PRACTICE OF BE}

$\mathrm{BE}$ is an essential drug product standard for both innovator and generic products. For innovator products, $\mathrm{BE}$ is used to establish therapeutic equivalence between the commercialized, marketed product and the clinical-scale product that underwent phase III safety and efficacy testing. The pivotal phase III studies that establish the evidence for the label indication(s), use(s), and dosing require that the clinically tested phase III product show evidence of bioavailability. All subsequent products that contain the same "drug," or "active pharmaceutical ingredient," and label must be bioequivalent to that clinically tested product. For example, additional $\mathrm{BE}$ evidence on the innovator product is required when the product undergoes various scale-up or postapproval changes (see, e.g., FDA SUPAC guidances ${ }^{15}$ ). Therefore, BE is the essential continuing standard for ensuring the therapeutic interchangeability and efficacy of pharmaceutical products.

However, the question of what scientific evidence is necessary to ensure $\mathrm{BE}$ is still much discussed. The in vivo $\mathrm{BE}$ approach has limits in its applicability; for example, in vivo studies in healthy subjects are difficult to justify when product changes can be considered "minor" (e.g., changes in color) or a drug has significant adverse effects (e.g., cancer and immune therapeutics). The 2010 European Medicines Agency BE guideline highlights this issue in its discussion of the "BCS-Based Biowaiver." ${ }^{\text {BCS }}$ has provided a sound scientific basis for deciding when in vivo tests should be required and when in vitro tests are sufficient or superior to in vivo testing for $\mathrm{BE}$. The BCS approach has established that, on the basis of current scientific understanding of the dissolution and gastrointestinal processes that oral drug products undergo, in vivo tests should not be required for certain drugs with certain drug product characteristics (e.g., high-permeability, highsolubility drugs in products that dissolve rapidly). ${ }^{2}$ Current FDA practice related to BCS biowaivers is limited to class I (high-permeability, high-solubility) drug substances. The 2010 European Medicines Agency Bioequivalence Guideline ${ }^{6}$ further extends its discussion of biowaivers to class III (low-permeability, highsolubility drugs) as well. In both cases, it is essential that the product demonstrate certain in vitro dissolution characteristics, i.e., rapid or very rapid dissolution. ${ }^{6}$ For drug products with the aforementioned permeability and solubility characteristics, we believe that a scientifically sound dissolution test in fact provides superior evidence of $\mathrm{BE}$ because, in in vivo testing, variations in systemic drug levels reflect mainly gastric and intestinal transit variations, obscuring product differences. ${ }^{3}$

\section{PERMEABILITY, METABOLISM, AND ABSORPTION}

Benet and Larregieu ${ }^{1}$ are particularly concerned with the regulatory use of the term "high permeability": "We believe that using extent of absorption (a thermodynamic measure) and intestinal permeability (a kinetic measure) interchangeably is 
not scientifically sound and is ambiguous from a regulatory perspective." In addition, they write, "An alternative surrogate for a high extent of absorption could be a high extent of metabolism" (high fraction metabolized $\left(\mathrm{F}_{\text {met }}\right)$ ). On the second point we agree, because for a drug to be metabolized it must (usually) be absorbed (although some 30 drugs have been shown to be metabolized by bacterial enzymes in the intestine). ${ }^{16}$ As for the first point, it is clear that Amidon et al. (1995) ${ }^{3}$ and the 2000 FDA $\mathrm{BCS}$ guidance ${ }^{2}$ use the fraction dose absorbed in humans $\left(\mathrm{F}_{\mathrm{abs}}\right)$ as the primary measure of intestinal permeability (of the entire gastrointestinal tract). This follows from mass balance analysis, in which the extent of absorption is assumed to be the drug lost from the intestine (assuming no chemical or enzymatic degradation in the gastrointestinal tract) by absorption into the body across the (mainly) epithelial cell membrane surface of the gastrointestinal tract. We define oral absorption as transport of the drug into the "first" biological tissue (the mucosal cells or other cells lining the GI tract or passing the tight junctions into the interstitial fluid). Systemic availability is often reduced by metabolism in the intestinal and liver tissue (after absorption), i.e., first-pass metabolism. The permeability of the whole gastrointestinal tract is, in general, dependent on the location in the tract and the time elapsed, given cellular differentiation along the gastrointestinal tract, upregulation and downregulation of cellular and membrane components, and the changing luminal environment. Experiments with Caco-2 cell monolayers cannot reflect this in vivo complexity.

The use of the terms "kinetic" and "thermodynamic" to describe measures for permeability and extent of absorption also needs to be clarified. Permeability is measured as a velocity (units $\mathrm{cm} / \mathrm{s}$ ) on the order of $1 \times 10^{-4} \mathrm{~cm} / \mathrm{s}$ (ref. 17) for a typical oral drug in the human jejunum. Extent of absorption (like extent of reaction) is a measure of absorption (conversion to product) in a definite time period (gastrointestinal transit time). It is not a thermodynamic value, because the in vivo state is clearly not one of equilibrium. The use of the term "high permeability" in this regulatory context is therefore defined by $\mathrm{F}_{\mathrm{abs}}$ in the context of the FDA guidance, ${ }^{2}$ although it is not specifically stated as a definition in the guidance. The guidance also suggests alternative methods for determining high permeability that do not require experiments in humans (e.g., using animal or tissue culture instead) when scientifically validated.

Benet and Larregieu refer explicitly to permeability as measured in the commonly used Caco- 2 in vitro cell monolayer system. Although this system can be used for many mechanistic studies of epithelial cell transport processes, it clearly does not represent the full range of differentiation of the epithelial cells, other cell types, and mucin that line the entire gastrointestinal tract. Although the variability of permeability determined in tissue culture ${ }^{18}$ under varying experimental conditions requires careful consideration, ${ }^{19,20}$ published results to date ${ }^{14,18,21}$ support the use of cell monolayer results for determining high permeability (no known false-positives). The FDA BCS guidance ${ }^{2}$ clearly states that the suitability of a permeability-assessment method must be demonstrated ${ }^{2}$ when using the method for BCS classification. The use of results pertaining to Caco-2 (or other) cell monolayers, along with other supporting results such as pharmacokinetic, physicochemical, and estimation methods in human subjects, ${ }^{22}$ will support a biowaiver application. Clearly, given the in vivo complexity, the terms "(intestinal) permeability" and "high permeability" must be used carefully and in the appropriate scientific context. ${ }^{23}$

Studies of $\mathrm{F}_{\mathrm{abs}}$ and $\mathrm{F}_{\text {met }}$ in humans vary in complexity depending on the metabolic and excretion pathways of the particular drug and its metabolites. ${ }^{16,24}$ The 2000 FDA BCS guidance uses $\mathrm{F}_{\mathrm{abs}}$ in humans as the principal basis for classifying a drug as having "high permeability." The guidance does offer alternative approaches to the determination of high permeability. These approaches can be used to classify a drug or drug product and would require additional information on the drug or drug product. Tissue culture results-for example, from the Caco-2 or other tissue culture systems - might not be sufficient in themselves to enable classification of a drug for regulatory or other purposes unless high permeability is suitably demonstrated. Where sufficient information from multiple sources is available (e.g., human pharmacokinetic and mass balance data), however, further human studies become unnecessary for establishing $\mathrm{BE}$, and additional human studies do not add any useful scientific information.

We therefore argue that both $\mathrm{F}_{\text {abs }}$ and $\mathrm{F}_{\text {met }}$ should be used as evidence on which to determine whether a drug or drug product should be classified as having "high permeability." It is important to understand how $\mathrm{F}_{\mathrm{abs}}$ and $\mathrm{F}_{\mathrm{met}}$ together provide the data necessary to classify a drug for BCS purposes, set a regulatory standard, and ensure that $\mathrm{BE}$ is based on an adequate range of evidence, including dissolution tests. Although it is true that considerable metabolism data on drugs are available, these data usually focus on metabolite and metabolism pathway identification. The data are usually semiquantitative and allow for only limited statistical analysis (small numbers of subjects). For example, Roffey et al. report, based on a survey, that the mean recovery of drug from mass balance studies in humans is $80 \%$ on average. ${ }^{24}$ When total dose can actually be accounted for, the fraction metabolized, along with other relevant data, can be used to classify a drug. However, it is important to note that, with the exception of gastrointestinal luminal and brush-border instability or metabolism, a drug must be absorbed in order to be metabolized. In the case of radiolabel studies involving both IV and oral dosing, both $\mathrm{F}_{\text {met }}$ and $\mathrm{F}_{\mathrm{abs}}$ can be determined (although usually with limited statistical evaluation). In the case of data from oral administration alone, however, the metabolites need to be identified ${ }^{6,16}$ before $\mathrm{F}_{\text {met }}$ and $\mathrm{F}_{\mathrm{abs}}$ can be determined. These complexities in the determination of drug metabolism demonstrate that absorption and metabolism should, where possible, be investigated and considered together. This would ensure greater reliability with regard to drug classification as a basis for setting regulatory standards for BE determination.

\section{PUBLIC POLICY}

Benet and Larregieu raise an additional public policy question regarding the confidentiality of biowaiver information. We acknowledge that these issues often form an undercurrent in the debates on BE. The regulatory policy regarding industry product proprietary information, market exclusivity, and the open availability of regulatory information and related decisions has 
significant implications for the costs of health care. BE policy also has substantial implications for the pharmaceutical industry and for the commercial development of new chemical entities. Our scientific view of this issue is that a scientifically sound BE dissolution test, developed and set on the basis of the clinically tested phase III product, would serve as the fundamental BE standard for all subsequent products that are pharmaceutically identical ${ }^{4}$ to the clinically tested product. We further agree with Benet and Larregieu $^{1}$ that this dissolution test should be made publicly available. However, we believe that it should be made publicly available only after regulatory approval and the expiration of the appropriate patent term and exclusivity periods. The term of exclusivity and of confidentiality of this proprietary BE dissolution methodology needs further discussion. However, this test could be developed on a new chemical entity product at the last stages of drug development, after attrition of the many unsuccessful drug candidates. Alternatively, it could be developed by regulatory authorities (e.g., by the FDA, the US Pharmacopeia, or the European Pharmacopoeia) while the innovator drug product is being marketed and under exclusivity, and it could then be made public after the patent and market exclusivity periods expire. This would allow the setting of scientific standards that would ensure the continued long-term clinical performance of a drug product and reduce the need for expensive and complex testing in human subjects. We stress that our view is that a scientifically sound $\mathrm{BE}$ dissolution test could be more routinely applied to oral drug products to ensure performance when certain clearly defined (e.g., ref. 15) changes to a drug product are to be made or when commercial development or manufacturing requires changes outside approved specifications (e.g., unavailability of an excipient). This BE dissolution test would be applicable worldwide for establishing the continued efficacy of this oral drug product.

\section{CONCLUSION}

BCS, with its focus on solubility, permeability, and absorption, provides a scientific basis for setting strong BE standards for oral drug products. Biopharmaceutics and Drug Disposition Classification System and metabolic data based on human studies are also useful to the BE regulatory decision process. Both types of information are included in a new drug application and may or may not be published and publicly available. The $\mathrm{F}_{\text {met }}$ value, when used with a clear understanding of the significant metabolic pathways, can be considered being equivalent to the $\mathrm{F}_{\mathrm{abs}}$ value. Advances in the science of $\mathrm{BE}$ have revealed that both in vitro and in vivo data are significant in the setting of a scientifically sound BE regulatory policy and standard. Although in vivo studies in humans are extensively used to document $\mathrm{BE}$, they should no longer be viewed as the only type of evidence that can be used to establish the BE of oral drug products.

\section{ACKNOWLEDGMENTS}

G.L.A. gratefully acknowledges the Alexander von Humboldt Foundation and NIH GM 037188 for support of this work.

\section{CONFLICT OF INTEREST}

The authors declared no conflict of interest.

(c) 2011 American Society for Clinical Pharmacology and Therapeutics
1. Benet, L.Z. \& Larregieu, C.A. The FDA should eliminate the ambiguities in the current BCS biowaiver guidance and make public the drugs for which BCS biowaivers have been granted. Clin. Pharmacol. Ther. 88, 405-407 (2010).

2. US Food and Drug Administration. Waiver of In Vivo Bioavailability and Bioequivalence Studies for Immediate-Release Solid Oral Dosage Forms Based on a Biopharmaceutics Classification System <http://www.fda.gov/ downloads/Drugs/GuidanceComplianceRegulatorylnformation/Guidances/ ucm070246.pdf> (2000).

3. Amidon, G.L., Lennernäs, H., Shah, V.P. \& Crison, J.R. A theoretical basis for a biopharmaceutic drug classification: the correlation of in vitro drug product dissolution and in vivo bioavailability. Pharm. Res. 12, 413-420 (1995).

4. US Food and Drug Administration. Approved Drug Products With Therapeutic Equivalence Evaluations (Orange Book) < http://www.accessdata.fda.gov/ scripts/cder/ob/default.cfm>pp. 1-3 (2010).

5. Chen, M.L. et al. Bioavailability and bioequivalence: an FDA regulatory overview. Pharm. Res. 18, 1645-1650 (2001).

6. European Medicines Agency Committee for Medicinal Products for Human Use. Guideline on the Investigation of Bioequivalence < http://www.ema. europa.eu/docs/en_GB/document_library/Scientific_guideline/2010/01/ WC500070039.pdf> (2010).

7. Carpenter, D. Reputation and Power: Organizational Image and Pharmaceutical Regulation at the FDA (Princeton University Press, Princeton, NJ, 2010).

8. Drug Bioequivalence. Recommendations from the Drug Bioequivalence Study Panel to the Office of Technology Assessment, Congress of the United States. J. Pharmacokinet. Biopharm. 2, 433-466 (1974).

9. Castle, W.B., Astwood, E.B., Finland, M. \& Keefer, C.S. White paper on the therapeutic equivalence of chemically equivalent drugs. JAMA 208, 1171-1172 (1969).

10. Tobbell, D.A. Allied against reform: pharmaceutical industry-academic physician relations in the United States, 1945-1970. Bull. Hist. Med. 82, 878-912 (2008)

11. US Food and Drug Administration. Guidance for Industry: Bioavailability and Bioequivalence Studies for Oral Administered Drug ProductsGeneral Considerations <http://www.fda.gov/downloads/Drugs/ GuidanceComplianceRegulatoryInformation/Guidances/ucm070124.pdf> (2003).

12. Yu, L.X. et al. Biopharmaceutics classification system: the scientific basis for biowaiver extensions. Pharm. Res. 19, 921-925 (2002).

13. Wu, C.Y.\&Benet, L.Z. Predicting drug disposition via application of BCS: transport/ absorption/ elimination interplay and development of a biopharmaceutics drug disposition classification system. Pharm. Res. 22, 11-23 (2005).

14. Chen, M.L. \&Yu, L. The use of drug metabolism for prediction of intestinal permeability (dagger). Mol. Pharm. 6, 74-81 (2009).

15. US Food and Drug Administration. Guidance for Industry: Immediate Release Solid Oral Dosage Forms Scale-Up and Postapproval Changes: Chemistry, Manufacturing, and Controls, In Vitro Dissolution Testing, and In Vivo Bioequivalence Documentation <http://www.fda.gov/downloads/Drugs/ GuidanceComplianceRegulatoryInformation/Guidances/ucm070636.pdf> (1995).

16. Sousa, T., Paterson, R., Moore, V., Carlsson, A., Abrahamsson, B. \& Basit, A.W. The gastrointestinal microbiota as a site for the biotransformation of drugs. Int. J. Pharm. 363, 1-25 (2008).

17. Lennernäs, H. Intestinal permeability and its relevance for absorption and elimination. Xenobiotica. 37, 1015-1051 (2007).

18. Sun, D. et al. Comparison of human duodenum and Caco-2 gene expression profiles for 12,000 gene sequences tags and correlation with permeability of 26 drugs. Pharm. Res. 19, 1400-1416 (2002).

19. Volpe, D.A. Variability in Caco-2 and MDCK cell-based intestinal permeability assays. J. Pharm. Sci. 97, 712-725 (2008).

20. Volpe, D.A. Application of method suitability for drug permeability classification. AAPS J. 12,670-678 (2010).

21. Yang, Y., Faustino, P.J., Volpe, D.A., Ellison, C.D., Lyon, R.C. \&Yu, L.X. Biopharmaceutics classification of selected beta-blockers: solubility and permeability class membership. Mol. Pharm. 4, 608-614 (2007).

22. Takagi, T., Ramachandran, C., Bermejo, M., Yamashita, S., Yu, L.X. \& Amidon, G.L. A provisional biopharmaceutical classification of the top 200 oral drug products in the United States, Great Britain, Spain, and Japan. Mol. Pharm. 3, 631-643 (2006).

23. Dahan, A. et al. High-permeability criterion for BCS classification: segmental/ pH dependent permeability considerations. Mol. Pharm. (2010); e-pub ahead of print 3 September 2010.

24. Roffey, S.J., Obach, R.S., Gedge, J.I. \& Smith, D.A. What is the objective of the mass balance study? A retrospective analysis of data in animal and human excretion studies employing radiolabeled drugs. Drug Metab. Rev. 39, 17-43 (2007). 